\title{
Drell-Yan physics at COMPASS
}

\author{
Catarina QUINTANS ${ }^{* \dagger}$ \\ LIP - Lisbon \\ E-mail: catarina.quintansecern.ch
}

\section{on behalf of the COMPASS Collaboration}

The COMPASS experiment at CERN will start in October 2014 its Drell-Yan (DY) physics program. This is the first transversely polarized target Drell-Yan measurement ever performed, and it will provide new insight to the transverse momentum dependent (TMD) parton distribution functions.

COMPASS measured already azimuthal single spin asymmetries in semi-inclusive DIS (SIDIS) processes, contributing in a decisive way to the present knowledge of the nucleon TMDs. The predicted sign change of the Sivers and Boer-Mulders TMDs when accessed from SIDIS or from DY processes will be verified by this new DY measurement.

XXII. International Workshop on Deep-Inelastic Scattering and Related Subjects 28 April - 2 May 2014

Warsaw, Poland

* Speaker.

${ }^{\dagger}$ A footnote may follow. 


\section{Introduction}

The COMPASS experiment at CERN takes data since 2002, to address a vast range of physics topics, among which the transversity parton distribution function (PDF) and the transverse momentum dependent (TMD) PDFs extraction. If both longitudinal and transverse momentum of partons inside the nucleon are considered, at leading twist 8 TMD PDFs of quarks are needed to describe the nucleon.

COMPASS has published results on the azimuthal spin asymmetries of hadrons produced in semi-inclusive deep inelastic scattering of muons in a transversely polarized proton target [1], namely observing a non-zero Sivers asymmetry for positive hadrons, and compatible with zero for the negative ones. This result, while qualitatively compatible with the HERMES experiment one [2], suggests an important $Q^{2}$ dependence of the Sivers TMD, that has been widely discussed recently [3].

The polarized Drell-Yan process gives an alternative access to TMD PDFs, with the advantage over SIDIS that no fragmentation functions are involved, the measured spin asymmetries relating to convolutions of 2 TMD PDFs. In leading order QCD, when having a transversely polarized target, the Drell-Yan cross-section can be written in a form that highlights these azimuthal asymmetries, see equation 1.1.

$$
\begin{aligned}
\frac{d \sigma}{d^{4} q d \Omega} & =\frac{\alpha^{2}}{F q^{2}} \hat{\sigma}_{U}\left\{\left(1+D_{\left[\sin ^{2} \theta\right]} A_{U U}^{\cos 2 \phi} \cos 2 \phi\right)\right. \\
& +\left|\vec{S}_{T}\right|\left(A_{U T}^{\sin \phi_{S}} \sin \phi_{S}+D_{\left[\sin ^{2} \theta\right]}\left(A_{U T}^{\sin \left(2 \phi+\phi_{S}\right)} \sin \left(2 \phi+\phi_{S}\right)\right.\right. \\
& \left.\left.\left.+A_{U T}^{\sin \left(2 \phi-\phi_{S}\right)} \sin \left(2 \phi-\phi_{S}\right)\right)\right)\right\}
\end{aligned}
$$

The 4 azimuthal asymmetries give access to the following convolutions: the Boer-Mulder TMD from pion with Boer-Mulders from proton for $A_{U U}^{\cos 2 \phi}$; the unpolarized TMD from pion with Sivers TMD from proton for $A_{U T}^{\sin \phi_{S}}$; the Boer-Mulders TMD from pion with pretzelosity TMD from proton for $A_{U T}^{\sin \left(2 \phi+\phi_{S}\right)}$; and the Boer-Mulders TMD from pion with transversity TMD from proton for $A_{U T}^{\sin \left(2 \phi-\phi_{S}\right)}$.

Due to their naive time reversal odd nature, Sivers and Boer-Mulders TMD PDFs change sign when accessed from DY or from SIDIS. The experimental observation of this sign-change is considered a crucial test of the TMD approach of non-perturbative QCD.

The COMPASS experiment has the unique opportunity to measure, with basically the same spectrometer, azimuthal spin asymmetries from transversely polarized Drell-Yan. The versatility of the COMPASS beam line allows to have hadron beams in the momentum range 50 to $280 \mathrm{GeV} / \mathrm{c}$. Using a negative pion beam of $190 \mathrm{GeV} / \mathrm{c}$ momentum, one will have u-quark dominance of the Drell-Yan process. Four azimuthal spin asymmetries will be measured, which are expected to be sizable in the valence quark region which corresponds to the COMPASS phase space. In figure 1 the phase space coverage of the 2 COMPASS measurements, in terms of $Q^{2}$ versus $x$ is shown. For SIDIS the data taken in 2010 with proton target [1] is presented, while the phase space for Drell-Yan in the mass range $4<M_{\mu \mu}<9 \mathrm{GeV} /^{2}$ is resulting from a full Monte-Carlo simulation. 


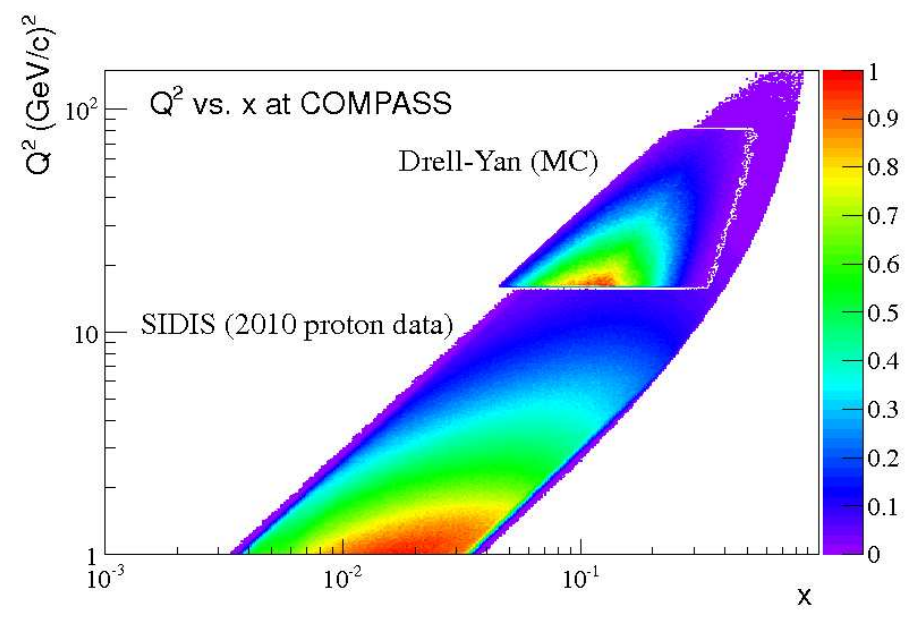

Figure 1: Phase space coverage of the SIDIS and Drell-Yan COMPASS measurements.

\section{Drell-Yan setup}

The COMPASS detector consists of a 2-stage spectrometer, allowing to measure low momentum and large emission angle particles, as well as high momentum particles emitted at small angles. The aperture of acceptance for muons is $\pm 180 \mathrm{mrad}$. The polarized target system uses the dynamic nuclear polarization method to align the spins of protons in an ammonia target placed in a strong magnetic field (a $0.6 \mathrm{~T}$ dipole field). The very low temperature of the target material, in the order of $60 \mathrm{mK}$, leads to extremely long relaxation time of the polarization (thousands of hours). The detailed description of the COMPASS setup can be found in [4].

For the Drell-Yan measurement of COMPASS, a negative pion beam of $190 \mathrm{GeV} / \mathrm{c}$ momentum will be used.The target will consist of 2 target cells, with $55 \mathrm{~cm}$ length and $2 \mathrm{~cm}$ radius each, and spaced by $20 \mathrm{~cm}$. As used in the past COMPASS SIDIS measurements, the target material will be solid state ammonia $\left(\mathrm{NH}_{3}\right)$. Using a dipole field to keep the proton spins in the target transversely polarized with respect to the pion beam direction, one can reach up to $90 \%$ polarization, with a dilution factor (which refers to the fraction of polarizable material in the target) of $22 \%$. The 2 target cells are oppositely polarized, and spin reversal is performed every few days, in order to minimize some of the systematic errors in the azimuthal spin asymmetries extraction.

In order to have meaningful statistics, the relatively low cross-section of the Drell-Yan process must be compensated by a high intensity pion beam. The maximum beam intensity achievable in COMPASS will be used, $10^{8}$ particles per second, the limit being dictated by the beam line itself, the allowed radiation dose in the hall, and to avoid local heating of the target (that could compromise the target polarization). In order to keep the detectors occupancy at reasonable level below 5\%, a thick hadron absorber will be placed immediately downstream the polarized target. The absorber design was optimized having in mind the highest hadrons stopping power, while keeping the multiple scattering of muons as low as possible. An absorber made of aluminum and alumina $\left(\mathrm{Al}_{2} \mathrm{O}_{3}\right)$, with a downstream $20 \mathrm{~cm}$ layer of steel, was chosen. In its inner part, the absorber contains a core of tungsten designed to stop the beam which did not interact in the polarized target. This so-called beam plug is in itself a heavy nuclear target useful to study the non-polarized Drell- 


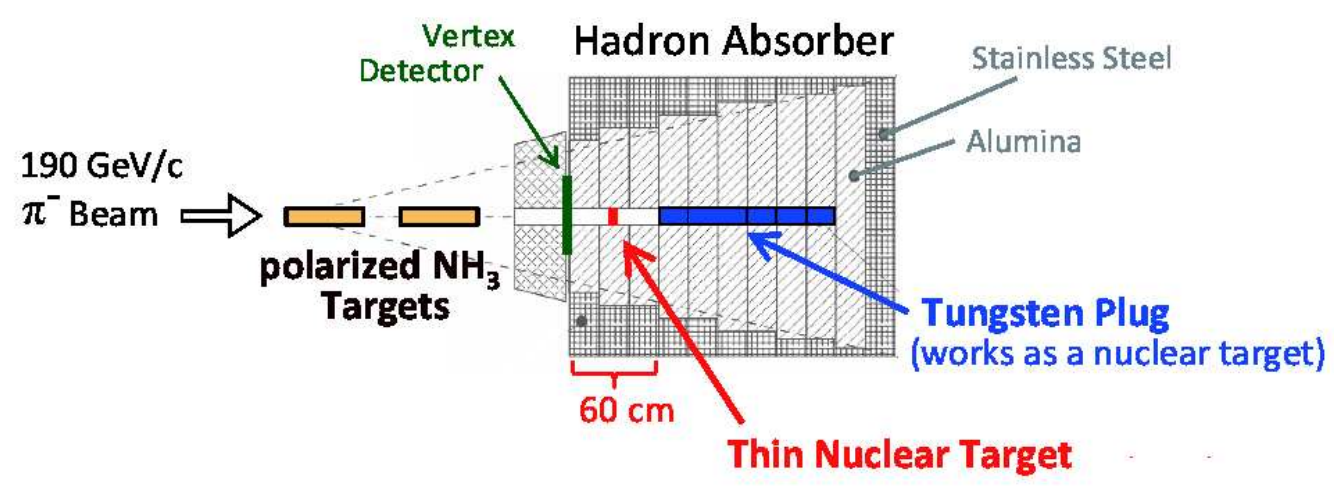

Figure 2: The target region and absorber of the COMPASS Drell-Yan experiment.

Yan process. An additional thin nuclear target of a lighter material, like carbon or aluminum, will be placed half way between target and beam plug. The data from these 2 unpolarized targets will allow to study the EMC effect in the Drell-Yan process, namely to investigate possible flavor dependence. Figure 2 shows the target region of the COMPASS Drell-Yan setup.

Due to the presence of the hadron absorber, the spectrometer will detect mostly muons. Nevertheless, a non-negligible punch-through of particles produced in the absorber itself will populate namely the first chambers. The dimuon mass spectrum will contain a combinatorial background of muons coming from pion and kaon decays, but this will not extend up to the region of interest for the Drell-Yan studies, the $4<M_{\mu \mu}<9 \mathrm{GeV} / \mathrm{c}^{2}$ mass range. Other sources of background are the open-charm semi-leptonic decays, but also in this case their contribution to the high masses can be neglected.

A dedicated dimuon trigger will be used, based in the coincidence of hodoscope signals. The trigger matrices guarantee the coincidences obeying homothety of the horizontal scintillator slabs in each pair of hodoscopes, as well as target pointing capability (the pointing is approximate, given the width of the slabs, allowing to trigger on dimuons from both the polarized target and the unpolarized ones).

The COMPASS geometrical acceptance was evaluated from a full Monte-Carlo of Drell-Yan events with dimuon masses $4<M_{\mu \mu}<9 \mathrm{GeV} / \mathrm{c}^{2}$, generated in Pythia and passed in a Geant3 simulation of the spectrometer. The global acceptance is $39 \%$. Figure 3 shows the acceptance as a function of several relevant variables: the dimuon mass, transverse momentum, Feynman-x, $\cos \theta$ and $\phi$ angle in the Collins-Soper frame, and target spin $\phi_{S}$ angle.

\section{Results from beam tests}

The feasibility of the COMPASS measurement was checked by several beam tests. In a test performed in 2009, a prototype hadron absorber, made of concrete and steel, was used. The target consisted of 2 polyethylene cylinders, $40 \mathrm{~cm}$ long each, spaced by $20 \mathrm{~cm}$. Since the test lasted only 3 days, the collected statistics is not enough to look at the high mass Drell-Yan events. The average beam intensity was $8 \times 10^{6}$ pions per second, an order of magnitude below the nominal. The data recorded contains about $7000 \mathrm{~J} / \psi$ events originated from the polyethylene target. This result is in 

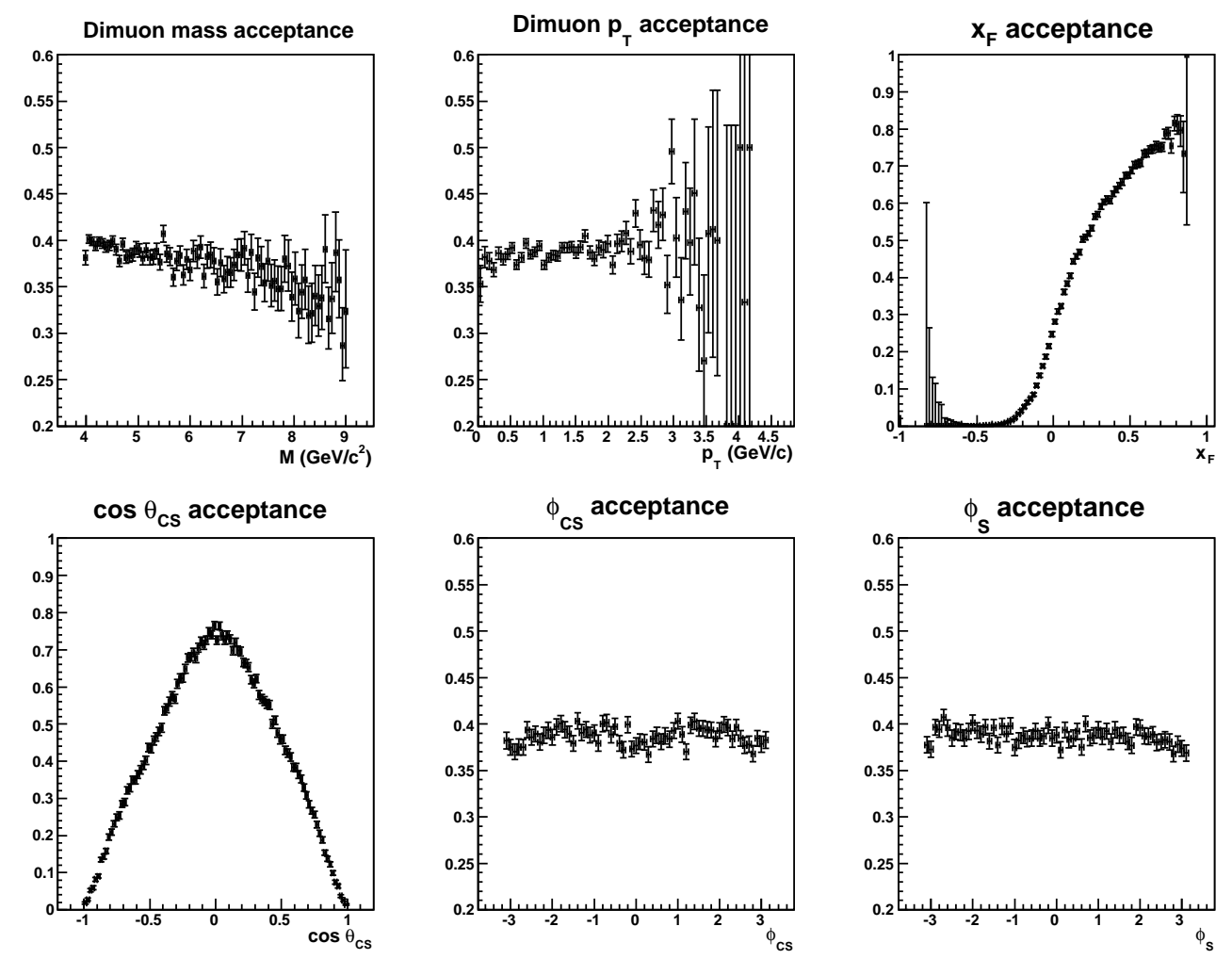

Figure 3: The dimuon geometrical acceptance of the COMPASS experiment, for Drell-Yan in the mass range $4<M_{\mu \mu}<9 \mathrm{GeV} / \mathrm{c}^{2}$.

good agreement with the expectation, given the low trigger efficiency. In fact, the dimuon trigger used was based on calorimetric signals, and had an estimated efficiency below 50\%.

Figure 4 shows the dimuon mass spectrum obtained, for events with dimuon trigger originated from the polyethylene target (right-hand side). The fitting function shown is the sum of 2 Gaussian functions (for $\mathrm{J} / \psi$ and $\psi^{\prime}$ ) and a decreasing exponential that effectively describes the background from combinatorial background, open-charm decays and Drell-Yan in the limited mass range fitted. Also shown on the left-hand side is the distribution of primary vertexes (vertexes containing at least 2 outgoing opposite charge muons and one incoming beam particle), where the clear separation between those originated from target cells and those from the tungsten beam plug is visible.

\section{Expected asymmetry accuracies}

The COMPASS Drell-Yan measurement will start in October 2014, and continue in 2015. In the anticipated 140 days of data collection, a total of 285 thousand Drell-Yan events in the mass range $4<M_{\mu \mu}<9 \mathrm{GeV} / \mathrm{c}^{2}$ will be accumulated, corresponding to a rate of approximately 2000 per day. This statistics is larger than the predicted in the COMPASS-II proposal [5] by a factor 2, thanks to the shorter SPS duty cycle, of 34 seconds (meaning that the $10^{8}$ particles/s will be available during 9.6 seconds in each 34 seconds). 

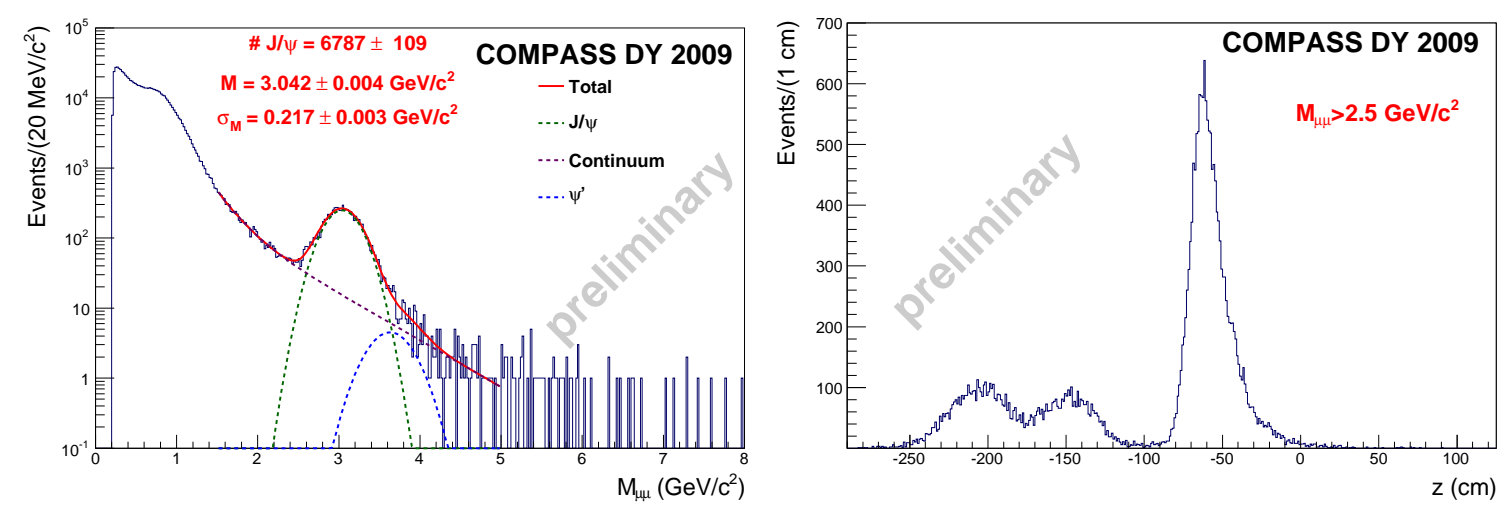

Figure 4: Dimuon mass distribution of triggered events originated from the polyethylene target cells (righthand side). The primary vertexes distribution of dimuon events originated both from the polyethylene target and the tungsten beam plug inside the absorber (left-hand side).

\begin{tabular}{|l|c|}
\hline $\begin{array}{l}\text { Asymmetry } \\
\text { uncertainties }\end{array}$ & $\begin{array}{c}\text { Dimuon mass }\left(\mathrm{GeV} / c^{2}\right) \\
4<M_{\mu \mu}<9\end{array}$ \\
\hline$\delta A_{U}^{\cos 2 \phi}$ & 0.005 \\
\hline$\delta A_{T}^{\sin \phi_{S}}$ & 0.013 \\
\hline$\delta A_{T}^{\sin \left(2 \phi+\phi_{S}\right)}$ & 0.027 \\
\hline$\delta A_{T}^{\sin \left(2 \phi-\phi_{S}\right)}$ & 0.027 \\
\hline
\end{tabular}

Table 1: Statistical uncertainties in the azimuthal asymmetries expected after 140 days of data collection.

Table 1 presents the expected statistical error for the 4 azimuthal asymmetries measured, assuming 140 effective days of data-taking. For the Sivers asymmetry the statistical error will be of the order of $1 \%$, possibly allowing a study in several bins of Feynman-x or transverse momentum.

The COMPASS Drell-Yan program could be extended to make measurements with polarized ${ }^{6} \mathrm{LiD}$ target, which would allow for flavor dependence studies of the TMDs. Also the use of a long unpolarized liquid hydrogen target would give valuable information for the Boer-Mulders TMD extraction. Collecting more data with the polarized ammonia target would provide precise characterization of the 4 asymmetries in bins of $x_{F}$ and $p_{T}$, and give access, for the first time, to the kaon TMD PDFs, profiting from the 2.5\% kaon contamination in the COMPASS negative hadron beam. All these studies could take place after the next SPS long shutdown, possibly after 2018, depending on the scientific committees approval.

\section{References}

[1] C. Adolph et al., COMPASS Collaboration, Phys. Lett. B 717 (2012) 383-389.

[2] A. Airapetian et al., HERMES Collaboration, Phys. Rev. Lett. 103 (2009) 152002.

[3] M. Aybat, A. Prokudin, T. Rogers, Phys. Rev. Lett. 109, 242003 (2012).

[4] P. Abbon et al., COMPASS Collaboration, Nucl. Instr. Meth. A 577 (2007) 455-518.

[5] COMPASS-II proposal, CERN-SPSC-2010-014, SPSCP-340, May 2010. 